\title{
Containing a Global Threat: The Virulence of Tuberculosis in Developed and Developing Countries
}

\author{
Lauren Skye Crawford \\ Department of Psychology, McGill University \\ 845 Sherbrooke St. W., Montréal, Québec, Canada \\ Tel: 1-514-398-6121Ｅ-mail: Lauren.Crawford@mail.mcgill.ca \\ Scott Nicholas Romaniuk \\ Department of International Relations, University of Aberdeen \\ Aberdeen, AB24 3QY, United Kingdom \\ Tel: 44-1224-272-726 E-mail: Scott.N.Romaniuk@gmail.com
}

Received: August 4, 2010 Accepted: August 18, 2010 doi:10.5539/gjhs.v3n1p77

\begin{abstract}
Pulmonary Tuberculosis (TB or tubercle bacillus) is increasing in global prevalence, and greatly threatens the populations of developed and developing countries. Although TB is often associated with developing countries, this communicable condition is currently on the rise in the United States, and elsewhere in the developed world. The proliferation of TB is a result of inadequacies or the non-existence of appropriate and decisively responsive intervention strategies and programs. Moreover, failure to employ adequate procedural response to diseases is heightened by insufficient knowledge of those afflicting communities globally. Practitioners are further challenged in the prevention of TB as a result of reported cases in developing countries being largely incomplete. Whereas one-third of the world's population, or two billion people are currently infected with the tubercle bacillus aerobic bacterium, it is expected that the number of TB-related deaths will rise from three million per year to five million by 2050 (World Health Organization, [hereafter, WHO], 2010).

Keywords: Communicability, Directly Observed Treatment Short-Course (DOTS), Epidemiological, Fluorescence Microscopy (FM), Millennium Development Goals (MDGs), Treatment, World Health Organization (WHO)
\end{abstract}

\section{Introduction}

According to the World Health Organization (WHO), the primary causes of illness and death in the developed world are cancer, and diseases of the respiratory, cardiovascular, and nervous systems (WHO, 2010). In the developing world, illness and death, and one of the greatest threats to human security is primarily the result of communicable diseases - infectious conditions transmitted from one individual to another either by direct contact or indirectly by fomites and vectors (WHO, 2010). These include, among others, respiratory infections, HIV/AIDS, infections at birth, diarrheal diseases as well as tropical diseases such as malaria. According to the World Watch Institute, all of the wars that have taken place during the twentieth century have resulted in approximately 1.1 million combatants and civilians per year (Worldwatch Institute, 2005). However, it is estimated that communicable diseases kill fourteen times that number of people annually (Worldwatch Institute, 2005). Although significant advances have been made in medical research, which has led health officials to declare victory in the campaign against infectious diseases, "old maladies such as tuberculosis, malaria, and cholera have spread geographically, and more than thirty previously unrecognized diseases such as Ebola, HIV, Hantavirus, and SARS have emerged as new threats to human well-being" (Worldwatch Institute, 2005).

Given this stark reality, it may safely be said that the burden of disease is global, and that the medical community's campaign against infectious diseases has resulted in failure rather than resounding success. Proliferation of such diseases is a result of inadequacies or the inexistence of appropriate and decisively responsive interventionist strategies and programs. Moreover, failure to employ adequate procedural responses 
to diseases is greatly heightened by insufficient knowledge of those afflicted communities globally.

This article addresses the issues and challenges in fighting pulmonary tuberculosis (TB or tubercle bacillus) in the developing world by presenting the argument that in order to employ effective prevention and treatment plans in high TB prevalent settings, we must rely on epidemiological evidence. In order to achieve this end, an overview of TB diagnostics will be presented in section two, followed by an overview of risk factors and causes in section three. Section four considers the complex constellation of TB transmission and progression taking into account transmission, probability, response, and the epidemiological burden of TB. Sections five and six analyze the existence of TB cases in various communities, and associated consequences of living with the disease. Following an overview of prevention and treatment solutions in section seven, the final section proposes a comprehensive strategy for fighting TB in developed and developing regions of the world.

\section{TB Diagnostics}

Due to similarities in symptoms, tests are critical to distinguish TB from lung cancer, lung abscesses, pneumoconiosis, and bronchitis (Neil, 2008). Multiple techniques exist that can be utilized to quantify the proportion of the population with latent or active TB, but due to their varying expenses, as well as ease of transport and administration, each method is differentially practical in unique community settings.

Symptom-screening is the initial way to assess TB risk. The definition of a TB suspect is based on coughing for three weeks or longer, as well as haemoptysis - coughing-up of blood or blood-stained sputum. Other symptoms include fever and night-sweats, which is identified in 77\% of patients with tuberculosis (Day, et al., 2006). When utilizing these criteria to assess the need for further laboratory investigation, sensitivity can be strengthened if two additional symptoms are included. In HIV-positive individuals, two further symptoms increases sensitivity from $48.8 \%$ to $79.1 \%$ (Ayles, et al., 2009).

The gold standard for TB testing is chest radiography, which will reveal lesions, cavities, scar tissue, and calcium deposits (Long, et al., 1997). This technique is often employed in tandem with smear-negative tuberculosis, but is a limited tool due to inter-observer variation and interpretation. Despite its restricted efficacy as a screening tool in and of itself, a retrospective review of routine public health program records in the Giang Province of Vietnam demonstrated that chest radiography might result in high yield and an affordable method for screening peoples living with HIV/AIDS (Shah, et al., 2008). Thus, given that chest radiography can increase the detection of other methods, it may be a useful tool in some settings.

Sputum smear microscopy is an additional practical diagnostic method to use in third world countries. The sputum smear is a smear obtained from the lower respiratory tract. Traditionally Ziehl-Neelsen (ZN) staining of sputum samples, also known as the acid-fast stain was used, but efforts have been made to improve the sensitivity of this earlier technique (Reid and Shah, 2009). Most protocols require that two separate cultures both test negative to rule out the possibility of tuberculosis. The preferred method to examine acid-fast bacilli such as microbacterium tuberculosis is to use Fluorescence Microscopy (FM). Since the technique of Fluorescence Microscopy increases the number of sputum samples that can be read in a span of time, it might be more cost effective than the traditional Ziehl-Neelsen approach (Tenover, et al., 1993). A cross-sectional study of TB suspects attending a clinic in Kenya compared the Ziehl-Neelsen and Florescence Microscopy techniques to analyze sputum specimens, and found that the Fluorescence Microscopy method using one or two specimens is more cost-effective and facilitates a shorter diagnostic process as compared to Ziehl-Neelsen (Kivihya-Ndugga, et al., 2003). This evidence counters the belief that Florescence Microscopy is too expensive for use in resource-limited countries (Tenover, et al., 1993).

The tuberculin skin test is the oldest test for tuberculosis and is especially useful in testing children. It is often used for initial screening in developed counties, although it is under-utilized in Africa and Asia. It employs a purified protein derivative that is injected under the top layer of skin on the inner forearm. Those individuals who have ever been exposed to TB will develop a red bump at the site of injection within two days. A red bump is an indication that their body has reacted to the antigens in the purified protein derivative (Nelson, 2007). Follow-up within 48-72 hours is necessary for test reading, and unfortunately, the test does not discriminate latent from active tuberculosis and simply indicates a TB infection. Of the two major tuberculin skin tests that exist at present, the Mantoux tuberculin skin test has widely replaced the Tine test because of its heightened reliability (Neil, 2008). A limitation is that it is commonly believed that prior Bacille Calmette-Guerin vaccination (BCG) interferes with the interpretation of a positive skin test (Nelson, 2007). Opposing these beliefs, a study performed in Bissau, Guinea-Bissau, investigated the risk factors for active TB, and found that the BCG vaccination in infancy or childhood only increased the likelihood of a positive skin test in individuals with household exposure to an active TB case (Gustafson, et al., 2007). Similarly to chest radiography, as a 
stand-alone detection tool, the tuberculin skin test is not sufficiently sensitive or specific. Nonetheless, it can be combined with other tests, and is a relatively inexpensive diagnostic tool (Reid and Sarita Shah, 2009).

The QuantiFeron-TB Gold test is a blood test that can detect infection and latent TB, and results are available in as little as 24 hours (Neil, 2008). A specific investigation of a population of undocumented immigrants that originated from high endemic TB in Brescia, northern Italy, illustrated that the QuantiFeron-TB Gold test is an effective confirmatory test for tuberculin skin testing positive individuals (Carvalho, et al., 2007). This blood test is especially useful because it is not subject to reader bias, and BCG inoculation does not affect the results (Neil, 2008).

A newly developed diagnostic test is the Interferon-Gamma Release Assay (IGRA) which is a T-cell based assay. This blood test can be more specific than tuberculin skin tests for diagnosing latent TB, but is still being examined. IGRAs are expensive and require a high level of experience to administer. In addition, little evidence exists to support their use in immuno-compromised patients or in disease-endemic counties (Reid and Sarita Shah, 2009).

Xpert MTB/RIF is an automated molecular test for Mycobacterium tuberculosis (MTB) and resistance to rifampin (RIF), endorsed by WHO in December 2010. Xpert MTB/RIF provides an accurate diagnosis in around 100 minutes, can be utilized outside of conventional laboratories, and is effective in the detection of the early TB, multi-drug-resistant pulmonary TB (MDR-TB), and HIV/TB co-infection (Boehme, et al., 2010). The test is predicted to increase the diagnosis of patients with drug-resistant TB three-fold, and HIV-associated TB two-fold. Further, the manufacturer (Cepheid) has agreed to reduce the market price of this test by $75 \%$ for countries with high TB burden. Although there are hopes that Xpert MTB/RIF will revolutionize TB care and control, it has yet to be widely incorporated in high TB prevalent settings (World Health Organization, 2010).

Taken together, there are many inadequacies of the available and older diagnostic tools, but they can still be valuable if they are optimally utilized. Thus, symptom-screening, blood tests, and a skin test have the capacity to detect TB infection in the latent stage, while chest radiographies, sputum microscopy, and Xpert MTB/RIF will detect the TB disease. In terms of use in resource-limited settings, symptom-screening can be used to screen for individuals who might have tuberculosis, but questionnaires are not standardized or all-inclusive. Hence, an improvement to symptom surveys would be to add more symptoms.

At this time, WHO strongly recommends that Xpert MTB/RIF should be used as the initial diagnostic test in individuals suspected of MDR-TB or HIV/TB, and conditionally recommends its use as a follow-on test to microscopy in settings where MDR-TB and or HIV is of lesser concern, especially in smear-negative specimens (World Health Organization, 2010). Major resource implications should also be recognized.

The Xpert MTB/RIF offers great promise for global TB diagnosis and care; however continued research is needed to expand the development and utilization of affordable, rapid, and sensitive tests that can be used in developing countries with minimal health care attendant training (Reid and Sarita Shah, 2009). Until this occurs, we must use current evidence to combine available screening tools for optimal TB detection.

\section{Risk factors and Causes}

There are multiple contributing factors to TB infection, but the highest incidence occurs in individuals who reside in crowded, poorly-ventilated, and unsanitary conditions. Contributing factors to the spread of TB include rapid population growth, poor case detection and cure rates in developing countries, and transmission in hospitals and other public places (Neil, 2008). The Center for Disease Control and Prevention (CDC) has outlined two categories to classify individuals at risk. These include individuals who have recently been infected with TB, and individuals with medical conditions that result in a weakened immune system.

Furthermore, homeless persons, smokers, those with multiple sex partners, injection drug users, substance abusers, individuals with low body weight, and individuals who work and reside in high TB-prevalent facilities such as hospitals, are at greater risk of TB (Center for Disease Control and Prevention, 2009). Therefore, much of the developing world is at high risk because populations in such regions are typically malnourished and/or immuno-suppressed (Long, et al., 2001). Non-occupational factors such as greater age - due to cumulative exposure to TB - and an education lower or equal to high school level are associated with having a positive interferon-gamma release assay (IGRA) (Lien, et al., 2009). Numerous studies that assess different risk factors have been published, and studies addressing some important items will be discussed as follows.

Crowding is a problem that is particularly relevant in developing countries. A case-control study in three counties in West Africa specifically looked at host-related and environmental risk factors in resource-poor countries. A further study that was performed in Lima, Peru, with similar results concluded that there was a 
strong association between public transportation for commuting and TB.

In addition, in Ethiopia, TB progression has been attributed to poor service delivery in public health facilities such as organization, management, and processes of TB medication treatment. Therefore, patients often have a limited general knowledge of TB, its treatment, causes, side effects, or indications for ceasing the medication treatment. A particular Ethiopian study demonstrated that patients whose treatment supervisors were not trained properly, were incapable of managing side effects, and who could not manage illnesses arising during treatment were less likely to complete their treatments. This indicates that poorly-trained and inadequately-supervised health care workers, as well as limited health facility hours of operation are related to high treatment interruption (Mesfin, et al., 2009).

In countries where there is stigma surrounding TB, such as in Ethiopia, awareness is essential. Stigma can play a detrimental role in early treatment and adherence to anti-TB medication (Mesfin, et al., 2009). For example, TB patients expressed feelings of anger and distress after original diagnosis of TB during focus group discussions in Pakistan. These negative emotions sometimes resulted in rejection of the diagnosis, and there were instances of patients claiming complete denial of the diagnosis and refusing the treatment. Therefore, stigmatization surrounding the infection can be an indirect causal factor.

\section{TB Transmission and Progression}

\subsection{Transmission}

TB is principally transmitted via an airborne route (Neil, 2008). However, some common misconceptions are that TB can spread by shaking someone's hand, sharing food or drink, or kissing, but these are not viable methods of transmission (Center for Disease Control and Prevention, 2009). Rather, people often contract TB when an infected individual sneezes, coughs, or speaks, whereby droplets containing the organism can linger in the air for several hours and may be inhaled. This means that contact is not required for contraction. If these infectious droplets are inhaled and reach the alveoli of the lungs, an individual will become infected (Neil, 2008).

\subsection{Probability}

The probability of TB transmission depends on a number of external and internal factors to the person of interest. Contraction depends on risk factors inherit to the person such as baseline health, in addition to extraneous factors like the concentration of the infectious droplet nuclei in the air, and the duration of exposure. However, after initial infection, few will typically progress to illness. Only approximately $2 \%$ of infected people will go on to develop active TB after exposure of one year, and 10\% of infected individuals will grow ill over the course of a lifetime. This is attributed to the fact that bacteria remain dormant in the human body and they become active only when the person grows older or becomes immuno-compromised (Neil, 2008). In the case where the infection is dormant, the infection will remain asymptomatic, and the person will have latent TB infection (Center for Disease Control and Prevention, 2009).

\subsection{Response}

When bacilli lodge into alveoli, an immune response will occur in the next six weeks and granulomas collection of immune cells - will form. This is the point in which a patient will test positive for TB and has the active form of the disease. Hospitalization is often required for individuals with the advance active form of TB, whereby, if possible, the patient should be placed in an isolated room to minimize airflow, and wear a mask. Bacterial count will lower in about two weeks of antimicrobial therapy, and the disease is then not longer infectious. It is at this point that the patient can be safely dismissed from the hospital (Neil, 2008).

\subsection{Epidemiological Burden of TB}

According to figures from the 2010 World Health Organization annual report on global TB control, in 2009, there were an estimated 14 million prevalent and 9.4 million incident cases worldwide, with $12 \%$ of new cases reported among HIV-positive individuals. Asia accounted for 55\% of global incident cases, while the African continent accounted for $30 \%$. Incidence rates were generally highest in Africa (greater than 300 cases per 100,000 people), with the exceptions of Myanmar, Cambodia and North Korea, and very high (100-299 cases per 100,000 people) in Russia, Central and South Asia, and most of South East Asia. As examples, in 2009, India had an estimated prevalence of 3 million TB cases, with 2 million incident cases and incident rate of approximately 100-299 cases per 100,000 people; Nigeria had 830,000 prevalent and 460,000 incident cases, with an incidence rate greater than 300 per 100,000 people; and Vietnam had a prevalence of 290,000 cases, an incidence of 180,000 cases and an incidence rate of roughly 100-299 cases per 100,000 people (World Health Organization, 2010). 


\section{Consequences of TB}

Of those infected with tuberculosis that do not receive treatment, about $5-10 \%$ of these individuals will develop TB disease some time in their lives, and those having a weaker immune systems will be at a higher risk of developing the disease (Center for Disease Control and Prevention, 2009).

In 2009, an estimated 1.3 million HIV negative people died from TB, and an estimated 400,000 million deaths TB deaths among HIV-positive individuals worldwide (World Health Organization, 2010). Specifically, HIV-positive deaths account for roughly $23 \%$ of TB global deaths annually (Center for Disease Control and Prevention, 2009).

A specific study preformed in the Philippines addressed the morbidity and mortality associated with TB. Data from several surveys over a four year period were analyzed, and it was established that over 500,000 disability adjusted life years (DALYs), which are years of life lost to premature mortality and time living in less than full health (World Health Organization, 2011), are lost due to illness and premature mortality every year in the Philippines. With regards to clinical outcomes, $28 \%$ of active TB patients are diagnosed and treated, $20 \%$ are never diagnosed and will die of the disease, and $6 \%$ will die after diagnosis due to inadequate care (Peabody, et al., 2005).

In addition to the mortality and morbidity contributes to tuberculosis, there are also social and personal consequences of having the disease. A focus group discussion study was carried out in Sialkot, Pakistan to explore the social stigmatization and general perception of TB. As a result of TB being viewed as a very dangerous, infectious, and incurable disease, and because of the inaccurate belief that TB is contagious even after a complete course of treatment, TB patients and their families endure stigmatization, social isolation, and rejection (Liefooghe, et al., 1995).

Social isolation by the community, family members, and friends was often found to continue much longer than the disease is medically considered contagious. Divorce as well as patients having difficulty finding partners, are two additional common indirect personal consequences of TB. This may partly stem from the misconception that pregnancy and TB treatment are incompatible, as this belief has been found to result in treatment noncompliance and relationship problems (Liefooghe, et al., 1995). Therefore, health providers should express a more supportive attitude and provide a good explanation about the disease at the time of disclosure in order to minimize inaccurate beliefs and associated stigma (Long, et al., 1997).

\section{TB in the Community: Zambia, Vietnam, and the Philippines}

In order to illustrate the severity of $\mathrm{TB}$, the prevalence in specific areas determined with varying measurement protocols will be further examined. Namely, communities in Zambia, Vietnam, and the Philippines are discussed herein. When a large community-based tuberculosis and HIV prevalence survey was administered in two sub-districts of Zambia, a prevalence ratio of 870 per 100,000 people was noted, with $36 \%$ of all TB attributable to HIV. Of interest, many individuals had culture positive tuberculosis without the necessary symptoms to meet the definition of a TB suspect. This indicates a need for a more sensitive screening process in Zambia (Ayles, et al., 2009).

The WHO has delineated Vietnam as one of the 22 WHO delineated high-burden TB countries (Lien, et al., 2009). In particular, a randomly selected population in Hanoi participated in a study aimed to assess the prevalence of TB. Methods such as X-rays, physical examinations, and questionnaires were utilized, and suspected active TB subjects underwent sputum smear microscopy. The sputum smear-positive prevalence ratio in Hanoi was measured to be 146 per 100,000 people for individuals fifteen years or older (Horie, et al., 2007).

The rapid population expansion in urban areas has resulted in urban settlements where there are widespread problems of poverty, overcrowding, poor hygiene, and sanitation. A specific study investigated the magnitude of tuberculosis in urban poor settlements in the Philippines. It was determined that the movement of the rural poor to the urban areas in search of a better life has resulted in a rapidly growing urban population that has overburdened health and sanitation facilities. The prevalence out of 10000 in the urban poor for active TB, culture positive TB, and smear positive TB were $60,14.8$, and 6.7 respectively. In the urban general population that was used as a comparison, the corresponding prevalence was 39,9, and 4.1. Therefore, TB in urban poor populations in the Philippines is significantly worse than that of the general urban population.

Another cross sectional study addressing the prevalence of tuberculosis among health workers in Vietnam concluded that in the city of Hanoi, $47.3 \%$ of hospital employees tested positive using Interferon-Gamma-Release-Assays (IGRA) (Lien, et al., 2009). Finally, nosocomial transmission, which is infection as a result of treatment in a hospital or heath care service unit for an unrelated condition, is particularly 
a problem in sub-Saharan Africa. Health care workers were studied from three hospitals in Kampala and Uganda, and a prevalence of 57\% latent TB and 1.7\% active TB was documented (Kayanja, et al., 2005). These high rates among health care workers illustrate the need for health facilities to contain the airborne bacteria by ensuring that the infected patient is isolated (Neil, 2008).

\section{Fighting TB: Prevention and Treatment Solutions}

The two major contributions to the progression of TB are delay in seeking treatment and abandoning treatment before completion. The key reasons for these behaviors include people's lack of knowledge and the implications of tuberculosis symptoms, inconvenience of travel to health care clinic, social stigma associated with TB, high cost of medication, organizational problems in proper follow up, and patient's perception of clinic facilities (Neil, 2008). Since the act of detecting and treating TB patients will have an immediate effect on TB prevalence and mortality, and by reducing transmission, we can decrease the incidence of the disease. A number of prevention and treatment solutions that have potential or have proved successful in some conditions will be discussed.

\subsection{BCG Vaccine}

The Bacille Calmette-Guerin (BCG) vaccine is an injection that has been prepared from a strain of the weakened live bovine tuberculosis bacilli. BCG has existed for over 80 years and is one of the most widely used of all existing vaccines (World Health Organization, 2009). According to the State of the World's Children 2009, 89\% of one-year-old children are immunized against TB with the BCG vaccine. This injection is given to children under two years of age to minimize the potential for advanced forms of TB, and is usually given at, or soon after birth. There appears to be consensus with regards to the protective effects of BCG in defending against disseminated tuberculosis - where the bacterial infection spreads from the lungs to other part of the body through the blood/lymph - but for protection against pulmonary tuberculosis where the lungs are chiefly involved, is not well understood (Pulickal and Fernandez, 2006).

The vaccine does not prevent primary infection and does not prevent reactivation of latent pulmonary infection (World Health Organization, 2009). In other words, the BCG vaccine is administered to prevent morbidity and mortality, and will therefore not eliminate TB. Due to these limitations, this vaccination is not effective in restricting TB transmission (Deeks, et al., 2005). The vaccine prevents the progression of the tuberculosis infection to the tuberculosis disease (Pulickal and Fernandez, 2006). Alternatives to the vaccine include screening and treatment of latent TB infection, and early detection and treatment of active TB. Unfortunately, these alternatives are expensive and require human resources (Deeks, et al., 2005).

The status of the protective efficacy of BCG in different studies varies with no effect found in rural or urban communities in South India (Bhatia, et al., 2004), and a moderate effect was found in Istanbul, Turkey, with a risk reduction of $24 \%$ (Soysal, et al., 2005). The World Health Organization previously recommended that a dose of the BCG vaccine should be given to all healthy infants in high burden TB countries, but due to recent evidence showing increased risk of disseminated BCG, the WHO no longer recommends HIV-infected infants to be immunized with the vaccine (Chokephaibulkit, et al., 2007). Some reported adverse effects of neonatal vaccination of BCG range from mild and localized complications to systemic or disseminated BCG disease in HIV-infected children (Hesseling, et al., 2006).

Use of the vaccine in the immune compromised may induce an infection caused by the BCG vaccine, and a local reaction may cause shedding of the organism, which can than go on to infect other immune compromised individuals (World Health Organization, 2009). For instance, a hospital-based surveillance study in the Western Cape Province of South African confirmed cases of disseminated BCG disease in children below the age of one. Nine children in their study were diagnosed with BCG disease and seven of these children were HIV infected. The researchers concluded that neonatal BCG vaccination may increase the risk for disseminated BCG disease to infants vertically infected with HIV by several hundred fold compared to HIV-negative infants (Hesseling, et al., 2006). On the other hand, additional studies, such as a retrospective study of Thai infants, have not found such complications from neonatal vaccination in HIV-infected children during infancy (Chokephaibulkit, et al., 2007).

For administration to HIV-negative infants, further researcher and development of a variety of vaccine types is necessary, such as one vaccine to prevent infection in infants, one to treat active $\mathrm{TB}$, and one to be offered to adults. Multiple vaccines will be required because it is doubtful that one vaccine will be able to do all of these tasks (World Health Organization, 2009). In the meantime, since the BCG vaccine has been proved efficacious for a number of years in infants with healthy immune systems, there should be continued avocation of the injection among this population worldwide. 


\subsection{Directly Observed Treatment-Short Course (DOTS)}

Directly Observed Treatment-Short course (DOTS) is central to TB prevention and treatment, and it involves having TB patients travel to a health care facility to receive their medications under the supervision of health care personnel that will ensure that medications are taken in the correct order and for the correct duration. Specifics regarding anti-TB medication that patients require for treatment will be discussed in detail in the next section (Neil, 2008). Since DOT ensures that people take every dose, the patients are only required to take the drugs two or three times per week (Neil, 2008). According to the WHO, DOTS consists of five main components: Government commitment, case detection through quality-assured bacteriology, standardized treatment with supervision and patient support, an effective drug supply and management system, and a standardized recording and reporting system allowing assessment of treatment (World Health Organization, 2009). These policies for TB control were put forward in 1993, and in 1997 the DOT component was added with the intent of improving treatment completion and avoiding multi-drug resistance TB (MDR-TB) (Gonzales, et al., 2004).

DOTS programs have been successful to varying degrees throughout the developing world, and several factors have been identified to improve upon. For example, the retrospective study in Kerala State, India found that with DOT 3\% versus $45 \%$ without DOT were likely to relapse (Balasubramanian, et al., 2000). In two districts of Tanzania, treatment success rate was $76.4 \%$ in those facilities that implemented DOTS (Mfinanga, et al., 2008). A cluster-randomized controlled trial compared community DOTS and family-member DOTS in Nepal, and the success rates were $85 \%$ and $89 \%$ respectively. These studies represent a sample of the research carried out on global DOTS program treatment outcomes, and in general, DOTS programs have improved the treatment success and reduced the transmission of Mycobacterium tuberculosis (Newell, et al., 2006).

The aim of DOTS includes two components; to encourage patients to continue treatment and to identify those who miss treatment, as well as preventing resistance to anti-tuberculosis drugs (Newell, et al., 2006). There are many methods of extending the implementation of DOTS into communities and to improve existing programs, and much can be learned from programs already in place. To be maximally effective, the DOTS program must be well functioning (Santha, et al., 2002), available and convenient, as well as confidential (Balasubramanian, et al., 2004).

Effective and well functioning TB prevention and treatment requires a health service with good management, proper diagnostic facilities, well-trained staff, and a consistent drug supply (Mfinanga, et al., 2008). In programs that are not performing optimally, most failures to treat patients are due to a failure of the program to ensure treatment, not failure of the treatment itself (Santha, et al., 2002). The likelihood that patients will complete their treatment depends largely on the existence of symptoms which usually disappear shortly after the start of treatment.

In order to ensure that TB suspects have access to diagnostic and treatment facilities, community involvement and expansion of public-private partnership in TB control is important. If private facilities take the initiative to run DOTS programs, they can increase the access of communities to TB programs. To illustrate, the availability of healthcare services that provide DOTS are still low in many areas, with only one-third of facilities offering TB laboratory services and one-fifth offering TB treatment services in northern Tanzania. Direct observation of treatment is not ideal in communities that have poor accessibility to health services because of the added inconvenience and cost associated with travel.

There is a need for greater human and financial resources allocated to health care facilities and laboratories, and governmental efforts to increase the community-based DOTS (Mfinanga, et al., 2008). Another alternative suggested by $\mathrm{WHO}$ is to have community volunteers observe treatment in remote communities (Newell, et al., 2006). There are also several other options and other individuals capable of performing DOT, including trained midwifes (Jagota, et al., 1998), members of non-governmental organizations (Chowdhury, et al., 1997), and the patient's family, which makes treatment for patients more convenient (Gonzales, et al., 2004). Undoubtedly, community-based treatment can also help decrease the chances for transmission between patients and health workers (Ahmad and Mokaddas, 2009).

In addition to availability of human resources to perform DOT, it has been reported that a further reason for non-participation of DOTS can be attributed to fear of social stigma and rejection which is often a larger concern for women than for men. For those in a Indian retrospective study DOTS program, more than one-quarter did not actually receive observed treatment, and reasons for this include advanced age and fear of social stigma. Therefore, perceived confidentiality is important in making people feel comfortable using the treatment (Balasubramanian, et al., 2000). 


\subsection{Antibiotic Treatment}

A number of antibiotics are effective against tuberculosis, and the typical length of treatment is usually six months or longer. This prolonged treatment, and the fact that the people must continue to adhere to medication after they feel well, has made adherence a major problem in TB treatment. The antibiotics that are most commonly administered include isoniazid, rifampin, pyrazinamide, and ethambutol. Although an estimated 95\% of those who take these drugs properly will be cured, each medication has a side effect, albeit typically minor (Nardell, 2008). Latent TB is treated with an antibiotic daily or twice a week for nine months, however patients with an impaired immune system may need multiple-medication therapy. Patients with active TB are treated with three to four medication combinations for two months, followed by two medications for another four to seven months (Neil, 2008).

The development of drug-resistant TB is the consequence of poor adherence to medical treatment, inappropriate prescription and irregular availability of medication, poor drug availability or mal-absorption, which is typical in HIV positive individuals, as well as poor medication quality. Resistance develops when bacterial growths susceptible to a medication are suppressed, but the drug-resistant mutants are not eliminated from the patient. Different strains of Multidrug-Resistant M. Tuberculosis (MDR-TB) are able to evolve due to sequential mutations in target genes, and these resistant bacilli can subsequently be transmitted to other people. These resistant strains can withstand the most-effective and first line anti-TB drugs. The treatment of MDR-TB requires more expensive second-line drugs which have more side effects (World Health Organization, 2009). The consequential rise in MDR-TB is hampering efforts to control and manage the infection, especially in developing countries (Ahmad and Mokaddas, 2009).

Some clinical indicators of MDR-TB include a fever several weeks after initial treatment, sputum that is positive 2-5 months after multi-drug regimen has been is prescribed, and deteriorating radiological parameters (Lalloo, et al., 2006). Treatment of the resistant bacilli involves a combination of drugs, some of which are highly toxic, not very successful, and are costly. Individuals with multi-drug-resistant TB must undergo prolonged treatment, and often experience high relapse rates, and are also at high risk for morbidity and mortality (Ahmad and Mokaddas, 2009). This long duration of treatment that typically spans anywhere from 18-24 months is a central contributor to the problem of adherence (Neil, 2008).

Multi-drug-Resistant Pulmonary TB (MDR-TB) is caused by strains of the TB bacteria that are resistant to at least the antibiotics including isoniad (INH) and rifampin (RIF) (Ahmad and Mokaddas, 2009). There are an estimated 424,000 MDR-TB cases every year with about 25,000 of these cases with extensively drug-resistant TB (XDR-TB). (World Health Organization, 2009). XDR-TB develops when second-line drugs used for the treatment of MDR-TB are misused or mismanaged and subsequently become ineffective. Treatment options are severely limited for patients with XDR-TB because these strains are resistant to first- and second-line drugs (World Health Organization, 2009). The World Health Organization has put forward the Global Plan to Stop TB, and set forward three major objectives, which aim to detect and treat 1.6 million MDR-TB cases by 2015 . However, few national TB programs have the facilities to perform drug-susceptibility testing (DST) for first-line drugs, let alone the testing of second-line drug resistance. Due to these inadequacies in health care services, only a mere $5 \%$ of MDR-TB cases are estimated to be detected. The achievements of these aims indicate a dire need for laboratories to obtain the instruments and human resources to detect first- and second-line DST with rapid methods (World Health Organization, 2009).

A general method used to optimize MDR-TB therapy is to use aggressive initial drug regimens. In addition to aggressive therapy, monitoring of sputum specimens once a month should be carried out until sputum culture conversion has been completed. In order to successfully manage patients with MDR-TB, treating centers must be equipped with rapid and accurate diagnostic procedures. Because HIV co-infected individuals already have a poor prognosis, their diagnosis is even more urgent (Ahmad and Mokaddas, 2009).

Treatment of MDR-TB in developing countries has been considerably less successful in comparison to developed countries due to the deficiency in rapid diagnostic methods, sputum culture and DST facilities, and access to second-line drugs (Ahmad and Mokaddas, 2009). DOTS-Plus is a management strategy that stems from and acts as a supplement to DOTS. DOTS-Plus is intended to be implemented in areas with moderate to high prevalence of MDR-TB to treat patients with drug resistant TB with second-line drugs. Some places where it is currently being employed include Mexico, Philippines, Haiti, and Kenya. Fortunately, the prices of second-line drugs have dropped significantly due to a partnership between the Working Group on DOTS-Plus and industry. This is making the drugs accessible to the poverty-stricken (WHO, 2009).

Taken together, there are a number of broad solutions that we must work towards including: (a) monitoring 
under DOTS with four first-line drugs should be incorporated for 6 months to decrease drug resistance, (b) rapid diagnosis and DST for first- and second-line drugs, (c) aggressive treatment in the initial treatment phase, as well as (d) increased laboratory support in developing countries. There are also a number of anti-TB drugs that are currently being developed, and hopefully they can continue to treat TB and MDR-TB patients (Ahmad and Mokaddas, 2009). The new development of Xpert MTB/RIF should increase the diagnosis of drug-resistant TB substantially in the developing world.

\subsection{TB Case Identification}

Due to the fact that latent tuberculosis can persist in a dormant mode for years, an infected individual will remain at risk for developing active TB, especially if their immune system becomes impaired. The Center for Disease Control and Prevention (CDC) recommends that screening and preventative measures must be aimed at high-risk groups for which the incidence of TB is higher than the general population. Priorities for screening must be set to help allocate resources and energy for those with the highest chances of transmission, morbidity, and mortality. This is because by screening low-risk persons, resources are being channeled away from treating those who are infected or those who would benefit from preventative therapy.

According to the CDC, individuals with active TB should be treated first, followed by those in contact with TB patients like health workers and family members, and finally those infected with latent TB and are at high risk for developing active TB - to prevent progression to the contagious disease - should be treated. The priorities for screening should be determined based on the assessment of existing resources and the amount of infection and disease in the individual community (Center for Disease Control and Prevention, 2009).

Health education involves motivating the patient to adopt behavior that is beneficial to their health. Most implementations assume that patients lack knowledge of their illness/treatment to adhere fully. Health education can be educational, where information is presented in a motivating manner, or it can behavioral where reward and reinforcement for appropriate behavior causes maintenance and habits. Finally, health education can be a combination of both the educational and behavioral approaches. This combined strategy was explored in two adjacent Cape Town health clinics in South Africa to test effects on adherence; the intervention significantly reduced the risk of patient non-adherence (Dick, 1997).

\subsection{Education as Preventive Action?}

Education concerning TB is important to minimize inaccurate information that can lead to stigma and to guide people so they may perform health behaviors that will minimize transmission and progression of TB and maximize their treatment. However, education is not sufficient to treat and prevent TB because case detection and having individuals report themselves to a health clinic when they show TB symptoms remains a major challenge.

Bangladesh Rural Advancement Committee (BRAC) is a national non-governmental organization that has relied on community health workers to detect and treat TB cases in their villages. Villagers were screened for the key symptom of a chronic cough, and individuals that tested positive for TB were given a short course of DOT. This program utilized monetary incentives for community health workers, and patients were asked to deposit a financial bond and sign an agreement to complete their treatment. The results were positive and the program was extended to a second and third phase.

A further method that has proved successful increasing detection of TB cases, increasing the performance of TB control, and in decreasing stigma associated with TB in a rural districts is the contribution of 'TB clubs,' in Ethiopia. These TB clubs consist of a number of TB patients living in the same area who all attend out-patient appointments on the same day together. A leader is elected who takes on a number of responsibilities. The leader is responsible for ensuring that all members attend their appointments, and they coordinate regular meetings in which patients can provide mutual support in adhering to treatment and have the opportunity to share personal experiences with side effects. Leaders will refer TB suspects in the community as well as report patients who are failing to make progress on medications and treatments.

\section{Conclusion}

This article has addressed the issues and challenges in fighting pulmonary tuberculosis in developing countries and the potential risk that is poses to developed counties as well. In doing so, the argument was presented that in order to employ effective prevention and treatment plans in high TB prevalent settings, reliance on epidemiological evidence must be significantly registered. Therefore, in areas where limited data exists, community-based surveys must be performed, and further methods to improve implementations and policies must be carried out (Ayles, et al., 2009). If researchers are able to obtain some of these answers, policy-makers 
and other agencies may have the means to efficiently decrease the clinical and economic burden of TB in highly affected areas, as well as prevent the proliferation of pulmonary tuberculosis in developed corridors of the world.

The possible elimination of TB will be particularly challenging in developing countries because of the necessity of working with restricted resources. Numerous methods that facilitate a decrease in TB occurrence were previously discussed, and there appears to be some general consensus amongst the multitude of primary studies preformed throughout the developing world. Some broad and inclusive objectives to which limited resources might be allocated towards include increasing case identification, expanding health clinic and community based DOTS to achieve greater compliance to treatment, and enhancing drug and laboratory availability.

\section{References}

Ahmad, S., Mokaddas, E. (2009). Recent advances in the diagnosis and treatment of multidrug-resistant tuberculosis. Respiratory $\quad$ Medicine, 1e14. doi:10.1016/j.rmed.2009.07.010, http://dx.doi.org/10.1016/j.rmed.2009.07.010

Ayles, H., Schaap, A., Nota, A., Sismanidis, C., Tembwe, R., De Haas, P., Muyoyeta, M., Beyers, N., Godfrey-Faussent, P. (2009). Prevalence of tuberculosis, HIV and respiratory symptoms in two Zambian communities: implications for tuberculosis control in the era of HIV. PLoS ONE, 4(5), e5602. doi:10.1371/journal.pone.0005602, http://dx.doi.org/10.1371/journal.pone.0005602

Balasubramanian, R., Garg, R., Santha, T., Gopi, P., Subramani, R., Chandrasekaran, V., Thomas, A., Rajeswari, R., Anandakrishnan, A., Perumal, M., Niruparani, C., Sudha, G., Jaggarajamma, K., Frieden, T., Narayanan, R. (2004). Gender disparities in tuberculosis: report from a rural DOTS programme in south India. International Journal of tuberculosis and lung disease, 8(3), 323-332.

Balasubramanian, V., Oommen, K., Samuel, R. (2000). DOT or not? Direct observation of anti-tuberculosis treatment and patient outcomes, Kerala State, India. International Journal for Tuberculosis and Lung Disease, 4(5), 409-413. <ncbi.nlm.nih.gov/pubmed/10815733>.

Basic TB Facts. (2009). Center for Disease Control and Prevention. <cdc.gov/tb/topic/basics/default.htm>.

Bhatia, V., Swami, H.M., Rai, S., Gulati, S., Verma, A., Parashar, A., Kumari, R. (2004). Immunization Status in Children. Indian Journal of Pediatrics, 71(4), 313-315. <springerlink.com/content/9375g05804108427/>.

Carvalho, A., Pezzolio, M., El-Hamad, I., Arce, P., Bigoni, S., Scarcella, C., Indelicato, A., Scolari, C.,

Boehme, C., Nabeta, P., Hillemann, R., Nicol, M., Shenai, S., Krapp, F., Allen, J., Tahirli, R., Blakemore, R., Rustomjee, R., Milovic, A., Jones, M. (2010). Rapid Molecular Detection of Tuberculosis and Rifampin Resistance. New England Journal of Medicine, 363(11), 1005-1015. $<$ nejm.org/doi/full/10.1056/NEJMoa0907847\#t=article> .

Chokephaibulkit, K., Chotpitayasunondh, T., Vanprapar, N., Waranawat, N., Mock, P., McConnell, M., Jetswang, B., Neeyapun, K., Tappero, J., Culnane, M. (2007). Assessment of Bacille Calmette-Guerin Vaccine Reaction in HIV-Exposed Thai Infants. Clinical Infectious Diseases, 45(8), 1016-1018. doi:10.1086/521929, http://dx.doi.org/10.1086/521929

Chowdhury, A., Chowdhury, S., Islam, N., Islam, A., Vaughan, P. (1997). Control of tuberculosis by community health workers in Bangladesh. The Lancet, 350 (9072), 169-172. $<$ inkinghub.elsevier.com/retrieve/pii/S0140-6736(96)11311-8>.

Chowdhury R., Chowdhury S., Islam, N., Islam, A., Vaughan P. (1997). Control of Tuberculosis by community health workers in Bangladesh. The Lancet, 350(9072), 169-172. doi:10.1016/S0140-6736(96)11311-8, http://dx.doi.org/10.1016/S0140-6736(96)11311-8

Day, H., Charalambous, S., Fielding, L., Hayes, J., Churchyard, J., Grant, D. (2006). Screening for tuberculosis prior to isoniazid preventative therapy among HIV-infected gold miners in South Africa. International Union Against Tuberculosis and Lung Disease, 10(5), 523-529. <ncbi.nlm.nih.gov/pubmed/16704034>.

Deeks, S., Clark, M., Scheifele, D., Law, B., Dawar, M., Ahmadipour, N., Walop, W., Ellis, E., King, A. (2005). Serious Adverse Events Associated with Bacille Calmette-Guerin Vaccine in Canada. The Pediatric Infectious Disease Journal, 24(6), 538-541. <ncbi.nlm.nih.gov/pubmed/15933565>.

Dick, J., Lombard, C. (1997). Shared vision- a health education project designed to enhance adherence to anti-tuberculosis treatment. International Journal for Tuberculosis and Lung Disease, 1(2), 181-186. $<$ ncbi.nlm.nih.gov/pubmed/9441085>.

Fortun, J., Martin-Davila, P., Navas, E., Perez-Elias, J., Cobo, J., Tato, M., Pedrosa, E., Gomez-Mampaso, G. 
(2005). Linezolid for the treatment of multidrug-resistant tuberculosis. Journal of Antimicrobial Chemotherapy, 56, 180-185. doi:10.1093/jac/dki148, http://dx.doi.org/10.1093/jac/dki148

Gonzales, R., Monroe, A., Assis, E., Palha, P., Villa, T., Netto, A. The performance of health services in providing Directly Observed Treatment (dot) in households to control tuberculosis. Revista da Escola de Enfermagem $d a \quad U \quad S \quad P, \quad 42(4), \quad 1-10 . \quad$ doi:10.1590/s0080-62342008000400003, http://dx.doi.org/10.1590/s0080-62342008000400003

Gustafson, P., Lisse, I., Gomes, V., Vieira, C., Lienhardt, C., Naucler, A., Jensen, H., Aaby, P. (2007). Risk Factors for Positive Tuberculin Skin Test in Guinea-Bissau. Epidemiology, 8(3), 340-347. doi:10.1097/01.ede.0000259987.46912.2b, http://dx.doi.org/10.1097/01.ede.0000259987.46912.2b

Hesseling A., Marais, B., Gie, R., Schaaf, H., Fine, P., Godfrey-Faussett, P., Beyers, N. (2006). The risk of disseminated Bacille Calmette-Guerin (BCG) disease in HIV-infected children. Vaccine, 25(1), 14-18. doi:10.1016/j.vaccine.2006.07.020, http://dx.doi.org/10.1016/j.vaccine.2006.07.020

Hesseling, A., Rabie, H., Marais, B., Manders, M., Lips, M., Schaaf, H., Gie, R., Cotton, M., van Helden, P., Warren, R., Beyers, N. (2006). Bacille Calmette-Guerin Vaccine-Induced Disease in HIV-Infected and HIV-Uninfected Children. HIV/AIDS, 42, 548-558. doi:10.1086/499953, http://dx.doi.org/10.1086/499953

Horie, T., Lien, L., Tuan, L., Tuan, P., Sakurada, S., Yanai, H., Keicho, N., Nakata, K. (2007). A Survey of Tuberculosis Prevalence in Hanoi, Vietnam. International Journal of Tuberculosis and Lung Disease, 11(5), 562-566. <ncbi.nlm.nih.gov/pubmed/17439682>.

Jagota, P., Chandrasekharan, S., Sumathi, G. (1998). Follow-up of pulmonary tuberculosis patients treated with short course chemotherapy through traditional birth attendants. Indian Journal of Tuberculosis, 45, 89-93. $<$ scholar.google.ca/scholar?cluster $=3221233676075995210 \& h l=e n \& a s \_s d t=2000>$.

Kayanja, H., Debanne, S., King, C., Whalen, C. (2005). Tuberculosis infection among health care workers in Kampala, Uganda. International Journal for Tuberculosis and Lung Disease, 9(6), 686-688. <ingentaconnect.com/content/iuatld/ijtld/2005/00000009/00000006/art00017>.

Kivihya-Ndugga, van Cleeff, M., Githui, W., Nganga, L., Kibuga, D., Odhiambo, J., Klatser, P. (2003). A comprehensive comparison of Ziehl-Neelsen and fluorescence microscopy for the diagnosis of tuberculosis in a resource-poor urban setting. International Journal of Lung Disease, 7(12), 1163-1171. $<$ ingentaconnect.com/content/iuatld/ijtld/2003/00000007/00000012/art00008>.

Lalloo, G., Naidoo, R., Ambaram, A. (2006). Recent advances in the medical and surgical treatment of multi-drug resistant tuberculosis. Current Opinion in Pulmonary Medicine, 12(3), 179-185.

Long, N., Johansson, E., Diwan, V., Winkvist, A. (2001). Fear and social isolation as a consequence of tuberculosis in Vietnam: a gender analysis. Health Policy, 58, 69-81.

Long, R., O’Connor, R., Palayew, M., Hershfield, E., Manfreda, J. (1997). Disseminated tuberculosis with and without a military pattern on chest radiography: a clinical-pathological-radiologic correlation. International Journal of Tuberculosis and Lung Disease, 1(1), 52-58. $<$ http://www.ingentaconnect.com/content/iuatld/ijtld/1997/00000001/00000001/art00010>.

Liefooghe, R., Michiels, N., Habib, S., Morgan, M., Muynck, A. (1995). Perception and Social Consequences of Tuberculosis: A Focus Group Study of Tuberculosis Patients in Sialkot, Pakistan. Social Science and Medicine, 41(12), 1685-1692. doi:10.1016/0277-9536(95)00129-U, http://dx.doi.org/10.1016/0277-9536(95)00129-U

Lien, L., Hang, N., Kobayashi, N., Yanai, H., Toyota, H., Toyota, E., Sakurada, S., Thuong, P., Cuong, V., Nanri, A., Mizoue, T., Matsushita, I., Harada, N., Higuchi, K., Tuan, L., Keicho, N. (2009). Prevalence and Risk Factors for Tuberculosis Infection among Hospital Workers in Hanoi, Viet Nam. PLoS ONE, 4(8), e6798. doi:10.1371/journal.pone.0006798, http://dx.doi.org/10.1371/journal.pone.0006798

Mesfin, M., Newell, J., Walley, J., Gessessew, A., Tesfaye, T., Lemma, F., Madeley, R. (2009). Quality of tuberculosis care and its association with patient adherence to treatment in eight Ethiopian districts. Health Policy and Planning, 24(6), 457-466. doi:10.1093/heapol/czp030, http://dx.doi.org/10.1093/heapol/czp030

Millennium Development Goals. Goal 6: Combat HIV/AIDS, malaria, and other diseases. (2009). United Nations Development Programme (UNDP). < undp.org/mdg/goal6.shtml $>$.

Mfinanga, G., Ngadaya, E., Kimaro, G., Mtandu, R., Lema, L., Basra, D., Lwila, F., Egwaga, S., Kitua, A. (2008). Capacity of healthcare facilities in the implementation of Direct Observed Treatment strategy for tuberculosis in Arumeru and Karatu districts, Tanzania. Tanzania Journal of Health Research, 10(2), 95-98. 
<ncbi.nlm.nih.gov/pubmed/18846787>.

Neil, J. (2008). Perioperative Care of the Patient with Tuberculosis. Association of periOperative Registered Nurses, 88(6). doi:10.1016/j.aorn.2008.08.013, http://dx.doi.org/10.1016/j.aorn.2008.08.013

Nelson, K. (2007). Tuberculin Testing to Detect Latent Tuberculosis in Developing Countries. Epidemiology, 18(3), 348-349. doi:10.1097/01.ede.0000259985.76928.64, http://dx.doi.org/10.1097/01.ede.0000259985.76928.64

Newell, J., Baral, S., Pande, S., Barn, D., Malla, P. (2006). Family-member DOTS and community DOTS for tuberculosis control in Nepal: cluster-randomized controlled trials.

Peabody, J., Shimkhada, R., Tan, C., Luck, J. (2005). The burden of disease, economic costs and clinical consequences of tuberculosis in the Philippines. Health Policy and Planning, 1-7. doi:10.1093/heapol/czi041, http://dx.doi.org/10.1093/heapol/czi041

Pulickal, A., Fernandez, J. (2006). Comparison of the Prevalence of Tuberculosis Infection in BCG Vaccinated versus Non-Vaccinated School Age Children. Indian Pediatrics, 44, 344-346. $<$ thelancet.com/journals/lancet/article/PIIS0140-6736(03)12950-9/fulltext $>$.

Reid, M., Sarita Shah, N. (2009). Approaches to tuberculosis screening and diagnosis in people with HIV in resource-limited settings. The Lancet, 9(3), 173-184. < doi:10.1016/S1473-3099(09)70043-X>.

Santha, T., Garg, T., Frieden, T., Chandrasekaran, V., Subramani, R., Gopi, P., Selvakumar, N., Ganapathy, S., Charles, N., Rajamma, J., Narayanan, P. (2002). Risk factors associated with default, failure, and death among tuberculosis patients treated in a DOTS programme in Tiruvallur District, South India, International Journal for $\begin{array}{lllll}\text { Tuberculosis and Lung } & \text { Disease, } & 6, & 780-788 .\end{array}$ <ingentaconnect.com/content/iuatld/ijtld/2002/00000006/00000009/art00007>.

Center for Disease Control and Prevention. (1995). Screening for Tuberculosis and Tuberculosis Infection in High-Risk Populations- Recommendations of the Advisory Council for the Elimination of Tuberculosis. Centre for Disease Control and Prevention <cdc.gov/mmwr/preview/mmwrhtml/00038873.htm>.

Shah, N., Anh, M., Thuy, T., Duong Thom, B., Linh, T., Nghia, D., Sy, D., Duong, B., Chau, L., Wells, C., Laserson, K., Varma, J. (2008). Population-based chest X-ray screening for pulmonary tuberculosis in people living with HIV/AIDS, An Giang, Vietnam. The Union, 12(4), 404-410. $<$ http://www.ncbi.nlm.nih.gov/pubmed/18371266>

Shin, S., Yagui, M., Ascencios, L., Yale, G., Suarez, C., Quispe, N., Bonilla, C., Blaya, J., Taylor, A., Soysal, A., Millington, K., Bakir, M., Dosanjh, D., Yasemin, A., Deeks, J., Efe, S., Staveley, I., Ewer, K., Lalvani, A. (2005). Effect of BCG vaccination on risk of Mycobacterium tuberculosis infection in children with household tuberculosis contact: a prospective community-based study. The Lancet, 366, 1443-1451. doi:10.1016/50140-6736(05)67534-4, http://dx.doi.org/10.1016/50140-6736(05)67534-4

Tenover, F., Crawford, J., Huebner, R., Geiter, L., Horsburgh, R., Good, R. (1993). The Resurgence of Tuberculosis: Is Your Laboratory Ready? American Society for Microbiology, 31(4), 767-770. $<$ ncbi.nlm.nih.gov/pmc/articles/PMC263557/>.

UNICEF. (2009). The State of the World's Children 2009: Maternal and Newborn Health. $<$ unicef.org/sowc09/report/report.php>.

Worldwatch Institute. (2005). Containing Infectious Disease. <worldwatch.org/node/3842>.

World Health Organization (WHO). (2009). Biologicals: BCG vaccine. $<$ who.int/biologicals/areas/vaccines/bcg/en/>.

----. (2009). Global Tuberculosis Control 2009: Epidemiology, Strategy, Financing. $<$ who.int/tb/publications/global_report/2009/en/index.html $>$.

----. (2010). Global Tuberculosis Control 2010: The Global Burden of TB. $<$ who.int/tb/publications/global_report/2010/en/index.html $>$.

----- (2011). Health statistics and health information systems: Global Burden of Disease (GBD). $<$ who.int/healthinfo/global_burden_disease/en/index.html $>$.

----. (2009). Initiative for Vaccine Research (IVR): BCG-the current vaccine for tuberculosis. $<$ who.int/vaccine_research/diseases/tb/vaccine_development/bcg/en/>.

----. (2009). Initiative for Vaccine Research (IVR): TB vaccines and vaccine development. $<$ who.int/vaccine_research/diseases/tb/vaccine_development/en/>. 
----. (2009). MDR/XDR-TB and other challenges: XDR-TB. < who.int/tb/challenges/xdr/en/index.html>.

---. (2010). Media Center: News 2010.

<who.int/mediacentre/news/releases/2010/tb_test_20101208/en/index.html $>$.

----. (2007). Review Guidelines the BCG vaccination of infants at risk of HIV infection. Bulletin of the World Health Organization, 3(82), 18-24. <who.int/entity/immunization/wer8221bcg_May07_position_paper.pdf>.

----. (2009). Tuberculosis (TB): Pursue high-quality DOTS expansion and enhancement. < who.int/tb/dots/en/>.

----. (2009). Tuberculosis (TB): Policy guidance on TB drug susceptibility testing (DST) of second line drugs (SLD). <who.int/tb/features_archive/xdr_mdr_policy_guidance/en/index.html $>$.

----. (2009). Tuberculosis (TB): DOTS-Plus and the Green Light Committee. $<$ who.int/tb/dots/dotsplus/management_old/en/>.

----. (2010). Tuberculosis (TB): infection and transmission. < who.int/mediacentre/factsheets/fs104/en/>. 